\title{
Extrusion-Based 30 Printing of Photocurable Hydrogels in Presence of Flavin Mononucleotide for Tissue Engineering
}

DOI: 10,17691 stm2018.10,1.11

Received November 15, 2017

A.G. Savelyev, PhD Student, Junior Researcher, Laboratory of Laser Biomedicine'; Junior Researcher,

Laboratory of Photonic Bioengineering2;

A.v. Sochilina, PhD Student, Laboratory "Polymers for Biology"3; Engineer-Researcher, Laboratory of Laser Biomedicine';

R.A. Akasov, PhD, Researcher, Laboratory of Photonic Bioengineering2; Researcher, Group of Biomedical Materials;

A.V. Mironov, PhD, Senior Researcher, Supercritical Fluid Technology Laboratory';

V.A. Semchishen, PhD, Leading Researcher, Laboratory for Nonlinear Optics of Surface and Laser-Plasma Processes';

A.N. Generalova, PhD, Senior Researcher, Laboratory "Polymers for Biology";

E.V. Khaydukov, PhD, Head of the Laboratory of Laser Biomedicine';

Head of the Laboratory of Photonic Bioengineering';

V.K. Popov, DSc, Head of the Supercritical Fluid Technology Laboratory'

'Federal Scientific Research Centre "Crystallography and Photonics", Russian Academy of Sciences, 59 Leninskiy Prospekt, Moscow, 119333, Russia;

2I.M. Sechenov First Moscow State Medical University, $8 / 2$ Trubetskaya St., Moscow, 119991, Russia;

${ }^{3}$ Shemyakin-Ovchinnikov Institute of Bioorganic Chemistry, Russian Academy of Sciences,

16/10 Miklukho-Maklaya St., Moscow, 117997, Russia

3D printing became a widely used technique for tissue engineering applications. This additive technology enables easy fabrication of very complicated structures. However, selection and preparation of initial compositions for 3D printing satisfying high biocompatibility and processability requirements still remains challenging. One of the most promising materials for mimicking of the living tissues are hydrogels possessing properties close to native tissues. In this work, the printability of hydrogels based on hyaluronic acid and poly(ethylene glycol) derivatives dissolved in phosphate buffer saline in presence of flavin mononucleotide as an endogenous photosensitizer has been studied. To produce a hydrogel pattern, the extrusion of photocurable composition has been combined with its simultaneous photoinduced crosslinking under laser irradiation at $450 \mathrm{~nm}$. Cytotoxicity of fabricated films and 3D scaffolds has been tested in vitro using human fibroblasts BJ-5ta.

Key words: extrusion; 3D printing; photocurable hydrogel; hyaluronic acid.

How to cite: Savelyev A.G., Sochilina A.V., Akasov R.A., Mironov A.V., Semchishen V.A., Generalova A.N., Khaydukov E.V., Popov V.K. Extrusion-based $3 \mathrm{D}$ printing of photocurable hydrogels in presence of flavin mononucleotide for tissue engineering. Sovremennye tehnologii v medicine 2018; 10(1): 88, https://doi.org/10.17691/stm2018.10.1.11

\section{Introduction}

Recent successes in regenerative medicine have made possible restoring of damaged tissues by assembling artificial mimicking constructs. Cartilage [1], bone [1, 2], blood vessels [3], skin [4], and liver [5] are only some examples of reproduced tissues that have been reported in the past decade. Fabrication of tissue engineering constructs usually begins with producing three-dimensional scaffolds representing a structural support for living cells. Mechanical properties, morphology, adhesion, and porosity of scaffolds can be varied in a wide range, depending on the mimicking tissue requirements. However, all materials for scaffolding as well as products of their biodegradation must be nontoxic both to the cells and surrounding tissues. One of the attractive candidates for those scaffolds are various hydrogels based on biocompatible macromolecules [6], such as hyaluronic acid and its derivatives [7], as hyaluronic acid (nonsulfated glycosaminoglycan) is one of the major native component of the human extracellular matrix for connective, epithelial, and neural tissues.

In this work hyaluronic acid has been modified to produce hyaluronic acid glycidyl methacrylate (HAGM)

Corresponding author: Alexander G. Savelyev, e-mail: a.g.savelyev@gmail.com 
which is capable of crosslinking initiated by light. Methacrylation of biocompatible hyaluronic acid enables wide possibilities for its successful implementing in 3D printing as a key component of photocurable composition (PCC), representing a water solution of photocrosslinkable macromolecules with a photoinitiator and/or photosensitizer. PCC can also include biofunctional molecules, catalysts, and other additional moieties. It is essential that hydrogels obtained as a result of PCC crosslinking possess properties close to those of living tissues. The precise control of mechanical and swelling properties of hydrogels can be performed by simple adjusting concentrations of precursors in the initial PCCs. In particular, the addition of small amount of nontoxic poly(ethylene glycol) diacrylate (PEGDA) [8] in PCC allows to decrease dramatically the unnecessarily high swelling ratio (SWR) of HAGM-based hydrogel $(S W R>2000 \%)$ and make it more elastic [9]. SWR of the sample is calculated according to the expression:

$$
\operatorname{SWR}(\%)=\frac{W_{s}-W_{d}}{W_{d}} 100 \%,
$$

where $W_{s}$ and $W_{d}$ are the weights of the swollen and dried hydrogels, respectively.

It is vitally important to provide the biocompatibility of all components of PCC for tissue engineering including photoinitiator and/or photosensitizer. Therefore, in the present work, riboflavin (vitamin B2) in its water-soluble form, flavin mononucleotide (FMN), has been proposed and used as an endogenous photosensitizer. Vitamin B2 non-toxicity and goodness as a dietary supplement have been approved by Food and Drug Administration. FMN possesses absorption in ultraviolet $A$ and nontoxic blue ranges of the spectrum [10]. Under irradiation FMN [11] can form either singlet oxygen via energy transfer to atmospheric oxygen or hydrogen peroxide and derivatives via radicalization [12]. All these reactive oxygen species are capable to activate the crosslinking process. Moreover, riboflavin photobleaching time exceeding 8 min [13] permits its effective implementation as a photosensitizer in the $3 \mathrm{D}$ printing process.

Herein we have developed a HAGM-based PCC containing FMN as a photosensitizer and small amount (ca. $5 \mathrm{wt} \%$ ) of PEGDA to optimize mechanical properties of the final hydrogel. The hydrogel scaffolds have been produced in the shape of lattices by extrusion-based 3D printing combined with simultaneous laser irradiation at $450 \mathrm{~nm}$. Scaffolds have been analyzed using optical microscopy. In vitro cytotoxicity tests of printed hydrogel structures have been performed using human fibroblasts BJ-5ta.

\section{Materials and Methods}

Chemical materials. Poly(ethylene glycol) diacrylate (PEGDA, $M_{n} \approx 575$ ), hyaluronic acid sodium salt (high molecular weight, ca. $5 \mathrm{MDa}$ ), glycidyl methacrylate, triethylamine, dimethylformamide, sodium azide, and acetone were purchased from Sigma-Aldrich (USA). Flavin mononucleotide (riboflavin-5'-phosphate) was obtained from Pharmstandard (Russia). Phosphate buffered saline (PBS) was purchased from Lonza (Switzerland). RPMI-1640 cell growth medium, L-glutamine, and penicillin-streptomycin (Pen Strep, 100x) were purchased from PanEko (Russia), fetal bovine serum (FBS) was from HyClone (USA).

Cell culture. Immortalized human fibroblasts BJ5 ta were grown in RPMl-1640 growth medium at $37^{\circ} \mathrm{C}$ in $5 \% \quad \mathrm{CO}_{2}$ humidified atmosphere. RPMI-1640 was supplemented with $10 \%$ FBS, 2 mM L-glutamine, $100 \mu \mathrm{g} / \mathrm{ml}$ streptomycin, and $100 \mathrm{U} / \mathrm{ml}$ penicillin. The medium was replaced every 3-4 days.

Photocurable composition preparation. Aiming to substitute sodium atoms in hyaluronic acid sodium salt by methacrylate units the reaction with glycidyl methacrylate was applied according to the method described in [14] and hyaluronic acid glycidyl methacrylate (HAGM) capable of crosslinking has been obtained as a result.

HAGM and PEGDA were placed in PBS containing FMN and subjected to sonication for $1 \mathrm{~h}$. Then this mixture was left overnight and sonicated again to produce a homogeneous solution. To avoid spontaneous crosslinking, preparation of PCCs was performed under yellow light conditions. The prepared PCCs contained 20 wt $\%$ of HAGM and $5 w t \%$ of PEGDA. The concentration of FMN was $0.01 \mathrm{wt} \%$.

Scaffold fabrication. Self-designed extrusion-based 3D printer has been used to produce experimental hydrogel structures. Initially, PCC was loaded in the syringe where the open end was fitted with $5 \mathrm{~mm}$ long $250 \mu \mathrm{m}$ inner diameter capillary. In order to start the extrusion, the plunger was pushed inside along the syringe. Plunger speed and positioning of the syringe in Z-direction, as well as the movement of the substrate in X-Y coordinates, were controlled using G-code in Repetier software. Acceleration parameters of plunger and translation stages were precisely adjusted in Repetier to ensure uniform thickness of extruded PCC layer deposited onto the substrate. Photocuring of the extruded PCC was performed by laser irradiation at $450 \mathrm{~nm}$ wavelength with intensity of $70 \mathrm{~mW} / \mathrm{cm}^{2}$. When the pattern was formed, the stage moved in Z-direction and the procedure was repeated. Inner structure and surface morphology of the scaffolds were analyzed by optical microscopy using HRM-300 microscope (Huvitz, Korea).

In vitro study. The cytotoxicity of the hydrogel samples was studied in the extract test as it was described earlier [15], and human fibroblasts BJ-5ta were used as model cells. Briefly, 3D printed hydrogel samples were incubated within RPMI-1640 cell culture medium supplemented with $10 \%$ of $\mathrm{FBS}$ at $37^{\circ} \mathrm{C}$ in $5 \% \mathrm{CO}_{2}$ humidified atmosphere, and the supernatants (extracts) were collected after $24 \mathrm{~h}$. The BJ-5ta fibroblasts were seeded into 96-well plate $\left(10^{4}\right.$ cells per well), and the plate was incubated overnight in $\mathrm{CO}_{2}$-incubator. Then the medium in each well was replaced with $100 \mu$ of the extracts. The cells 
cultivated in the medium without the extracts were used as a control. Cell viability was measured by a MTT assay, which is a colorimetric assay for assessing cell metabolic activity through the reducing of the tetrazolium dye 3-(4,5-dimethylthiazol-2-yl)-2,5-diphenyltetrazolium bromide to its insoluble formazan. For this reason, after $24 \mathrm{~h}$ incubation with extracts, an MTT solution $(0.5 \mathrm{mg} / \mathrm{ml})$ was added to the wells, and the cells were incubated at $37^{\circ} \mathrm{C}$ for $3 \mathrm{~h}$. Formazan crystals formed in the living cells were dissolved in $100 \mu$ of dimethyl sulfoxide, and an optical density was measured at $570 \mathrm{~nm}$ using a Multiscan plate reader (Flow Laboratories, USA). Relative cell viability $(\mathrm{V})$ was calculated according to the following expression:

$$
\mathrm{V}=\left(O D_{\text {test }}-O D_{\text {background }}\right) /\left(O D_{\text {contro }}-O D_{\text {background }}\right) 100 \% \text {, }
$$

where $\mathrm{OD}_{\text {test }}, \mathrm{OD}_{\text {control, }}$ and $\mathrm{OD}_{\text {background }}$ are optical densities of sample, control, and background, respectively.

To demonstrate cell growth on the hydrogel surface, printed samples were placed into the 24-well plates and seeded with BJ-5ta fibroblasts $(20,000$ cells per each sample), and successive gradual cell growth was observed within 7 days.

OHAUS Starter $3100 \mathrm{pH}$-meter (OHAUS, USA) was employed to control $\mathrm{pH}$ of solutions.

\section{Results and Discussion}

First, we performed methacrylation of hyaluronic acid to produce HAGM. The synthesized hyaluronic acid derivative combines advantages of its biocompatibility and capability to photocrosslinking. Therefore, we chose HAGM as the main component of PCC. In order to perform gelation of the entire volume of the initial multicomponent PCC after exposure process, the concentration of capable to photocrosslinking HAGM macromolecules must exceed the minimal threshold value of $16 \mathrm{wt} \%$ discussed in [9]. The formation of a disordered 3D polymer network threading through the entire exposed volume and triggering the appearance of elastic properties becomes possible only above this threshold concentration.

The water-soluble form of riboflavin was proposed as an endogenous photosensitizer in PCC. FMN used in our experiments is characterized by a broad UV-blue absorption band with two peaks illustrated in Figure 1 (left). Using FMN in HAGM-based PCCs makes possible photocuring at $450 \mathrm{~nm}$ wavelength. Additionally, in order to avoid the unnecessary high swelling of the final HAGMbased hydrogel in water solutions, we added linear polymer PEGDA to all PCCs. Thus, we prepared PCC for 3D printing containing $20 \mathrm{wt} \%$ of HAGM, $5 \mathrm{wt} \%$ of PEGDA $\left(M_{n} \approx 575\right)$ and 0.01 wt $\%$ of FMN dissolved in PBS.

The scheme of our custom-designed extrusion-based $3 \mathrm{D}$ printer is presented in Figure 1 (right). The printer was equipped with $450 \mathrm{~nm}$ continuous wave semiconductor laser source. Owing to the focusing lens attached to the end facet of laser the divergent laser beam could expose the currently printed point or the whole sample. Depending on the irradiated area the power of the semiconductor laser was adjusted to provide $70 \mathrm{~mW} / \mathrm{cm}^{2}$ intensity.

To demonstrate the possibilities of our 3D printer, we fabricated scaffolds in the shape of lattices with period $1.4 \mathrm{~mm}$ as shown in Figure 2 (left). The lattices were formed using HAGM-based PCC by sequential printing of 25 layers. The printing speed was adjusted to $0.3 \mathrm{~mm} / \mathrm{s}$. During the extrusion process, the printing area was continuously irradiated at $450 \mathrm{~nm}$ wavelength to produce gelation of hydrogel layer. Finally, when the scaffold had been printed, we applied the additional exposure for
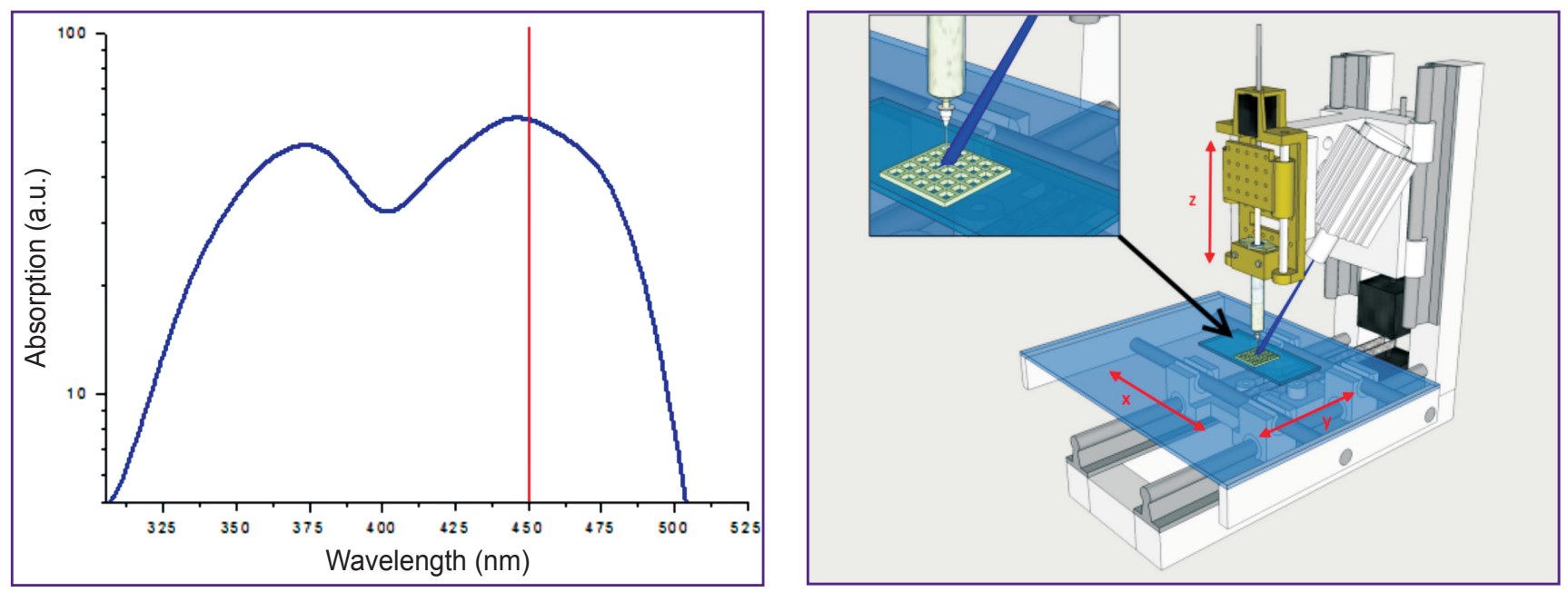

Figure 1. Absorption spectrum of flavin mononucleotide; the vertical line (left) indicates the blue light wavelength at $450 \mathrm{~nm}$. Scheme of extrusion-based 3D printing (right), the inset shows zoomed image of photocurable composition flow squeezing out of the nozzle and divergent laser beam 
Figure 2. Scaffold structure on the surface of cover glass produced by extrusion-based 3D printing with period of lattice $1.4 \mathrm{~mm}$ (left) and zoomed image of side view of the printed scaffold obtained with the optical microscope Huvitz with $50 \times$ objective (right)
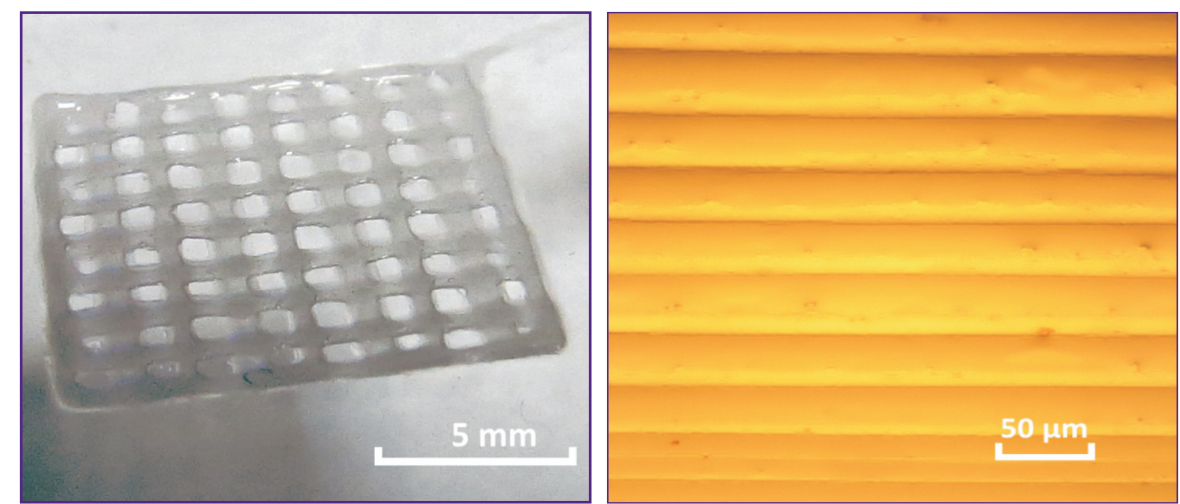

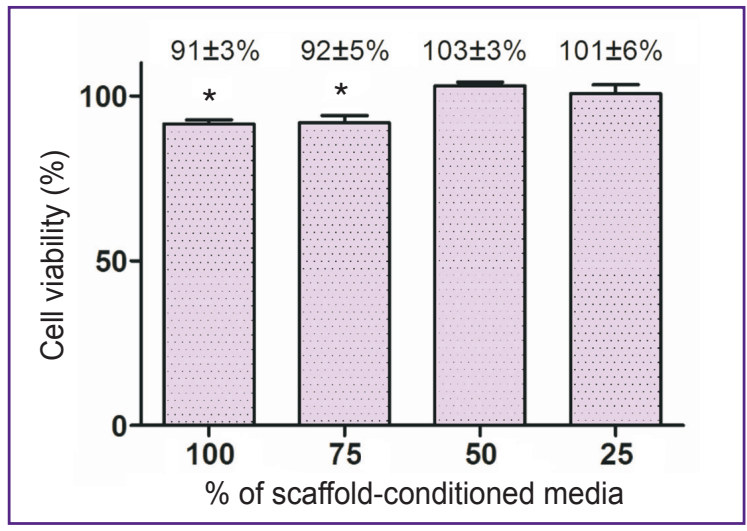

Figure 3. MTT assay data for the viability of BJ-5ta fibroblasts in the presence of hydrogel-conditioned media: $100 \%$ means hydrogel extract only; $75 \%$ means $75 \%$ of extract and $25 \%$ of non-conditioned RPMI- $1640 ; 50 \%$ means $50 \%$ of extract and $50 \%$ of non-conditioned RPMI-1640; $25 \%$ means $25 \%$ of extract and $75 \%$ of non-conditioned RPMI- 1640 . Nonconditioned RPMI-1640 medium was used as a control. The data are the mean $\pm S D$. P-values were calculated by MannWhitney $U$ test $\left({ }^{*}\right.$ indicates a $p$-value $<0.05$ )

10 min in order to uniform the degree of crosslinking in different hydrogel layers. Extrusion-based 3D printing of all structures was performed onto the smooth glass surface and the fabricated samples could be easily peeled out without destruction. Moreover, the scaffolds placed in PBS indicated stability for 3 weeks in PBS due to optimized swelling properties.

The surface of produced scaffolds was observed using optical microscope Huvitz with 50x objective (Huvitz, Korea). Figure 2 (right) illustrates the side wall of the scaffold. The wall represents uniform layered structure. The average height of every layer was measured as $30 \mu \mathrm{m}$. The total height of scaffolds was ca. $750 \mu \mathrm{m}$.

Prior to in vitro cytotoxicity study all printed samples were stored in PBS for 5 days to remove FMN and products of its photolysis from hydrogel structures.

The acute cytotoxicity of the hydrogel samples was evaluated using an extract test by MTT assay. It was found that hydrogel extracts slightly reduced the BJ-5ta cell growth (10-20\% slower in comparison to controls) (see Figure 3). To clarify the possible reason of cell growth deceleration, the $\mathrm{pH}$ measurements of the extracts, both in RPMI-1640 and in de-ionized water, were conducted. Since no $\mathrm{pH}$ changes were observed, we suppose that cytotoxic effect could be caused by spontaneous daylight photoactivation of remaining FMN following by reactive oxygen species and free radical production [16]. Nevertheless, the fibroblasts were able to grow on the surface of hydrogels, confirming high biocompatibility of the resulted samples.

\section{Conclusions}

The printability of a new HAGM-based hydrogel photocurable composition has been studied. The elastic lattice structures have been produced using extrusion-based 3D printing combined with photocuring by laser light at $450 \mathrm{~nm}$. We have demonstrated that human fibroblasts BJ-5ta were capable of attaching and growing on the surfaces of HAGM-based hydrogel scaffolds. Thus, we conclude that 3D printing of HAGMbased photocurable compositions containing flavin mononucleotide as an endogenous photosensitizer represents an attractive methodology for various tissue engineering applications.

Acknowledgments. This work was supported by the Federal Agency of Scientific Organizations (Agreement No.007-Г3/43363/26) in part of 3D printing and the Russian Science Foundation (Project No.17-19-01416) in part of PCC preparation.

Conflict of interest. The authors confirm the absence of financial and other conflicting interests that could influence their work.

\section{References}

1. Takato T., Mori Y., Fujihara Y., Asawa Y., Nishizawa S., Kanazawa S., Ogasawara T., Saijo H., Abe T., Abe M., Suenaga H., Kanno Y., Sugiyama M., Hoshi K. Preclinical and clinical research on bone and cartilage regenerative medicine in oral and maxillofacial region. Oral Science 
International 2014; 11(2): 45-51, https://doi.org/10.1016/ s1348-8643(14)00008-1.

2. Gu Y., Huang W., Rahaman M.N., Day D.E. Bone regeneration in rat calvarial defects implanted with fibrous scaffolds composed of a mixture of silicate and borate bioactive glasses. Acta Biomater 2013; 9(11): 9126-9136, https://doi. org/10.1016/j.actbio.2013.06.039.

3. Pankajakshan D., Agrawal D.K. Scaffolds in tissue engineering of blood vessels. Can J Physiol Pharmacol 2010; 88(9): 855-873, https://doi.org/10.1139/y10-073.

4. Zhong S.P., Zhang Y.Z., Lim C.T. Tissue scaffolds for skin wound healing and dermal reconstruction. Wiley Interdiscip Rev Nanomed Nanobiotechnol 2010; 2(5): 510 525, https://doi.org/10.1002/wnan.100.

5. Mazza G., Al-Akkad W., Telese A., Longato L., Urbani L., Robinson B., Hall A., Kong K., Frenguelli L., Marrone G., Willacy O., Shaeri M., Burns A., Malago M., Gilbertson J., Rendell N., Moore K., Hughes D., Notingher I., Jell G. Del Rio Hernandez A., De Coppi P., Rombouts K., Pinzani M. Rapid production of human liver scaffolds for functional tissue engineering by high shear stress oscillation-decellularization. Sci Rep 2017; 7(1): 5534, https://doi.org/10.1038/s41598-01705134-1.

6. He Y., Yang F., Zhao H., Gao Q., Xia B., Fu J. Research on the printability of hydrogels in 3D bioprinting. Sci Rep 2016 6(1): 29977, https://doi.org/10.1038/srep29977.

7. Kufelt O., El-Tamer A., Sehring C., Schlie-Wolter S., Chichkov B.N. Hyaluronic acid based materials for scaffolding via two-photon polymerization. Biomacromolecules 2014; 15(2): 650-659, https://doi.org/10.1021/bm401712q.

8. Mazzoccoli J.P., Feke D.L., Baskaran H., Pintauro P.N. Mechanical and cell viability properties of crosslinked low- and high-molecular weight poly(ethylene glycol) diacrylate blends. J Biomed Mater Res A 2010; 93(2): https://doi.org/10.1002/ jbm.a.32563.

9. Savelyev A.G., Bardakova K.N., Khaydukov E.V., Generalova A.N., Popov V.K., Chichkov B.N., Semchishen V.A. Flavin mononucleotide photoinitiated cross-linking of hydrogels: polymer concentration threshold of strengthening.
J Photochem Photobiol A Chem 2017; 341: 108-114, https:// doi.org/10.1016/j.jphotochem.2017.03.026.

10. Turunen S., Käpylä E., Terzaki K., Viitanen J., Fotakis C., Kellomäki M., Farsari M. Pico- and femtosecond laser-induced crosslinking of protein microstructures: evaluation of processability and bioactivity. Biofabrication 2011; 3: 045002, https://doi.org/10.1088/1758-5082/3/4/045002.

11. Khaydukov E.V., Mironova K.E., Semchishen V.A., Generalova A.N., Nechaev A.V., Khochenkov D.A., Stepanova E.V., Lebedev O.I., Zvyagin A.V., Deyev S.M., Panchenko V. Ya. Riboflavin photoactivation by upconversion nanoparticles for cancer treatment. Sci Rep 2016; 6: 35103, https://doi.org/10.1038/srep35103.

12. Agostinis P., Berg K., Cengel K.A., Foster T.H. Girotti A.W., Gollnick S.O., Hahn S.M., Hamblin M.R., Juzeniene A., Kessel D., Korbelik M., Moan J., Mroz P., Nowis D., Piette J., Wilson B.C., Golab J. Photodynamic therapy of cancer: an update. CA Cancer J Clin 2011; 61(4): 250-281, https://doi.org/10.3322/caac.20114.

13. Lin J. Analysis on the critical issues of UV light induced corneal cross linking. International Journal of Latest Research In Engineering and Computing (IJLREC) 2013; 1(1): 104-109.

14. Bencherif S.A., Srinivasan A., Horkay F., Hollinger J.O., Matyjaszewski K., Washburn N.R. Influence of the degree of methacrylation on hyaluronic acid hydrogels properties Biomaterials 2008; 29(12): 1739-1749, https://doi. org/10.1016/j.biomaterials.2007.11.047.

15. Drozdova M.G., Zaytseva-Zotova D.S., Akasov R.A., Golunova A.S., Artyukhov A.A., Udartseva O.O., Andreeva E.R., Lisovyy D.E., Shtilman M.I., Markvicheva E.A. Macroporous modified poly (vinyl alcohol) hydrogels with charged groups for tissue engineering: preparation and in vitro evaluation. Mater Sci Eng C Mater Biol Appl 2017; 75: 10751082, https://doi.org/10.1016/j.msec.2017.03.017.

16. Kamaev P., Friedman M.D., Sherr E., Muller D. Photochemical kinetics of corneal cross-linking with riboflavin. Invest Ophthalmol Vis Sci 2012; 53(4): 2360-2367, https://doi. org/10.1167/iovs.11-9385. 